\title{
A dense disk of dust around the born-again Sakurai's object ${ }^{\star} \star \star$
}

\author{
O. Chesneau ${ }^{1}$, G. C. Clayton ${ }^{2}$, F. Lykou ${ }^{3}$, O. De Marco ${ }^{4}$, C. A. Hummel ${ }^{5}$, \\ F. Kerber ${ }^{5}$, E. Lagadec ${ }^{3}$, J. Nordhaus ${ }^{6}$, A. A. Zijlstra ${ }^{3}$, and A. Evans ${ }^{7}$ \\ ${ }^{1}$ UMR 6525 H. Fizeau, Univ. Nice Sophia Antipolis, CNRS, Observatoire de la Côte d'Azur, Av. Copernic, 06130 Grasse, France \\ e-mail: olivier . chesneau@ob-azur.fr \\ 2 Department of Physics \& Astronomy, Louisiana State University, Baton Rouge, LA 70803, USA \\ 3 Jodrell Bank Centre for Astrophysics, The A. Turing Building, The Univ. of Manchester, Oxford Rd, Manchester M13 9PL, UK \\ 4 Department of Astrophysics, American Museum of Natural History, Central Park West at 79th Street, New York, NY 10024, USA \\ 5 European Southern Observatory, Karl-Schwarzschild-Strasse 285748 Garching bei München, Germany \\ 6 Department of Astrophysical Sciences, Princeton University, Princeton, NJ 08544, USA \\ 7 Astrophysics Group, Lennard-Jones Laboratories, Keele University, Staffordshire ST5 5BG, UK
}

Received 17 October 2008 / Accepted 18 November 2008

\section{ABSTRACT}

\begin{abstract}
Context. In 1996, Sakurai's object (V4334 Sgr) suddenly brightened in the center of a faint Planetary Nebula (PN). This very rare event was interpreted as being the reignition of a hot white dwarf that caused a rapid evolution back to the cool giant phase. From 1998 on, a copious amount of dust has formed continuously, screening out the star that remained embedded in this expanding high opticaldepth envelope.

Aims. We present observations that we use to study the morphology of the circumstellar dust to investigate the hypothesis that Sakurai's Object is surrounded by a thick spherical envelope of dust.

Methods. We acquired unprecedented, high angular-resolution, spectro-interferometric observations, with the mid-IR interferometer MIDI/VLTI, which resolved the dust envelope of Sakurai's object.

Results. We report the discovery of a unexpectedly compact $(30 \times 40$ milliarcsec, $105 \times 140$ AU assuming a distance of $3.5 \mathrm{kpc})$, highly inclined, dust disk. We used Monte Carlo radiative-transfer simulations of a stratified disk to constrain its geometric and physical parameters, although such a model is only a rough approximation of the rapidly evolving dust structure. Even though the fits are not fully satisfactory, some useful and robust constraints can be inferred. The disk inclination is estimated to be $75^{\circ} \pm 3^{\circ}$ with a large scale height of $47 \pm 7 \mathrm{AU}$. The dust mass of the disk is estimated to be $6 \times 10^{-5} M_{\odot}$. The major axis of the disk $\left(132^{\circ} \pm 3^{\circ}\right)$ is aligned with an asymmetry seen in the old PN which was re-investigated as part of this study. This implies that the mechanism responsible for shaping the dust envelope surrounding Sakurai's object was already at work when the old PN formed.
\end{abstract}

Key words. ISM: planetary nebulae: individual: Sakurai's object - stars: AGB and post-AGB - stars: circumstellar matter stars: mass-loss - techniques: high angular resolution - techniques: interferometric

\section{Introduction}

Sakurai's Object (V4334 Sgr), first detected in 1996, is the central star of a planetary nebula (CSPN), that experienced a Very Late Thermal Pulse (VLTP), which is a helium-shell flash while on the white-dwarf cooling track that can influence the late evolution of low-mass stars (Herwig 2001; Lawlor \& MacDonald 2003; Hajduk et al. 2005). The extended very faint planetary nebula (PN) bears witness to the previous evolution of this star, confirming that the latest large mass ejection during the PN phase occurred several thousands years ago (Kerber et al. 1999). This new "final flash", which began 12 years ago, returned the star very briefly to the Asymptotic Giant Branch (AGB) stage, explaining why these sources are often called "Born-again" objects. As such, a final flash is astronomically very brief (only a few tens of years), so that the observation of such an event is rare. Only the event experienced by V605 Aql

\footnotetext{
* Based on observations made with the Very Large Telescope Interferometer at Paranal Observatory under program 079.D-0415.

$\star \star$ Reduced visibilities and differential phases are available in electronic form at the CDS via anonymous ftp to

cdsarc.u-strasbg.fr (130.79.128.5) or via

http://cdsweb.u-strasbg.fr/cgi-bin/qcat?J/A+A/493/L17
}

can be considered as directly comparable (Clayton et al. 2006; Clayton \& De Marco 1997). V605 Aql underwent a final flash in 1917 and appears today to be still embedded in a disk-like dust structure (Clayton et al. 2006; Hinkle et al. 2008).

The analysis of the very rapid evolution of the spectral energy distribution (SED) of Sakurai's Object (Eyres et al. 1999; Kerber et al. 1999; Tyne et al. 2002; Käufl et al. 2003; Eyres et al. 2004; Evans et al. 2006; Worters et al. 2008) has suggested that the average dust grain size has been increasing and probably the rate of dust formation has been increasing as well. The results were based mainly on modeling of radiative transfer in a spherical dust shell. Tyne et al. (2002) suggested that the source was expanding and might be resolvable by 8-10 $\mathrm{m}$ class telescopes in the mid-IR. Kerber et al. (2002) discovered a fast outflow emerging from the source and suggested a bipolar morphology for the fast moving gas seen in optical spectra. Recently, it has been suspected that there is a strong asymmetry in the circumstellar shell around Sakurai's Object, but the dust shell itself had yet to be resolved in the optical or near-IR (Evans et al. 2006; van Hoof et al. 2007).

Observations using the Very Large Telescope Interferometer are presented in Sect.2. In Sect. 3 we derive some physical 
Table 1. Observing log.

\begin{tabular}{llccc}
\hline \hline OB & Time & Base & \multicolumn{2}{c}{ Projected baseline } \\
& & & $\begin{array}{c}\text { Length } \\
\text { [metre] }\end{array}$ & $\begin{array}{c}\text { PA } \\
\text { [degrees] }\end{array}$ \\
\hline Sak-1 & 2007-06-29T01 & U2-U3 & 41.9 & 14.6 \\
Sak-2 & $2007-06-29 T 02$ & U2-U3 & 45.9 & 37.1 \\
Sak-3 & $2007-06-29 T 06$ & U2-U3 & 44.4 & 48.4 \\
Sak-4 & $2007-06-30 T 01$ & U3-U4 & 51.3 & 101.0 \\
Sak-5 & $2007-06-30 T 06$ & U3-U4 & 54.7 & 124.3 \\
Sak-6 & $2007-06-30 T 08$ & U3-U4 & 39.2 & 159.5 \\
\hline
\end{tabular}

Calibrators: $\quad$ HD 152334 K4III $3.99 \pm 0.07$ mas, $\quad$ HD 163376 MOIII $3.79 \pm 0.12$ mas, HD 169916 K1III $3.75 \pm 0.04$ mas, HD 177716 K1III $3.72 \pm 0.07$ mas.

parameters of the dust shell using 2D radiative-transfer models, and discuss our results in Sect. 4.

\section{Observations}

The source was observed in June 2007 with MIDI (Leinert et al. 2003; Ratzka et al. 2007) the mid-IR recombiner of the Very Large Telescope (VLT). The MIDI/VLT Interferometer operates like a classical Michelson interferometer combining the midIR light ( $N$ band, 7.5-13.5 $\mu \mathrm{m}$ ) from two VLT Unit Telescopes (8.2 m, UTs). A typical MIDI observing sequence was followed, as described in Ratzka et al. (2007). MIDI provided single-dish acquisition images with a spatial resolution of about 250 mas at $8.7 \mu \mathrm{m}$, a flux-calibrated spectrum at low spectral resolution $(R=30)$, and 6 visibility spectra from the source. The observations were performed in High_Sens mode, implying that the photometry of the source was recorded subsequently to the fringes. The visibility errors range between $8 \%$ and $15 \%$. The accuracy of the absolute flux calibration is better than $10 \%$. The log of the observations is given in Table 1 . We used two different MIDI data reduction packages: MIA, developed at the Max-Planck-Institut für Astronomie, and EWS, developed at the Leiden Observatory (MIA + EWS ${ }^{1}$, ver.1.5.1).

The MIDI images show that the core is unresolved at $8.7 \mu \mathrm{m}$, implying that its angular diameter must be less than 150-200 mas. The mean level of the visibility spectra is low, indicating that the source is well resolved by MIDI/VLTI (typical scale $30 \times 40$ mas), but the spectral modulation implies that it is a complex source (see Fig. 2). Significant differential phases ( $\pm 10-20^{\circ}$ peak-to-peak) were observed for all baselines except Sak.4 and Sak.5 (Table 1), which exhibit phases close to zero $\left( \pm 5^{\circ}\right)$.

The data were complemented by two Spitzer/IRS spectra obtained in April 2005 and May 2007 (Evans et al. 2006; Evans et al. 2009, in preparation), and a continuum-subtracted [O III] image of the surrounding PN acquired in October 2002 with the instrument VLT/FORS1 (Hajduk et al. 2005). Figure 1 shows the Spitzer and MIDI spectra which are characterized by a steeply rising flux toward long wavelengths. The [OIII] image of the PN is shown in Fig. 3. The appearance of the nebula is almost circular, with weak but significant spatial structure.

\section{Physical parameters of the disk}

The large, spectrally-dependent variations of the MIDI visibilities could be interpreted as being double-point or ring structures.

1 Available at http://wWw.strw. leidenuniv.nl/ nevec/MIDI/ index.html
Table 2. Model parameters.

\begin{tabular}{ccc}
\hline \hline & Parameters & Parameter range \\
\hline$T_{\text {eff }}(\mathrm{K})$ & 12000 & - \\
Luminosity $\left(L_{\odot}\right)$ & 10000 & - \\
Distance $(\mathrm{kpc})$ & 3.5 & - \\
\hline Inclination $\left(^{\circ}\right)$ & 75 & 3 \\
PA angle $\left(^{\circ}\right)$ & 132 & 3 \\
Inner radius (AU) & 65 & 10 \\
Outer radius (AU) & 500 & No constraint \\
$\alpha$ & 2.0 & 0.1 \\
$\beta$ & 1.0 & 0.1 \\
$h_{100 \mathrm{AU}}(\mathrm{AU})$ & 47 & 7 \\
Dust mass $\left(M_{\odot}\right)$ & $6 \times 10^{-5}$ & $3 \times 10^{-5}$ \\
\hline
\end{tabular}

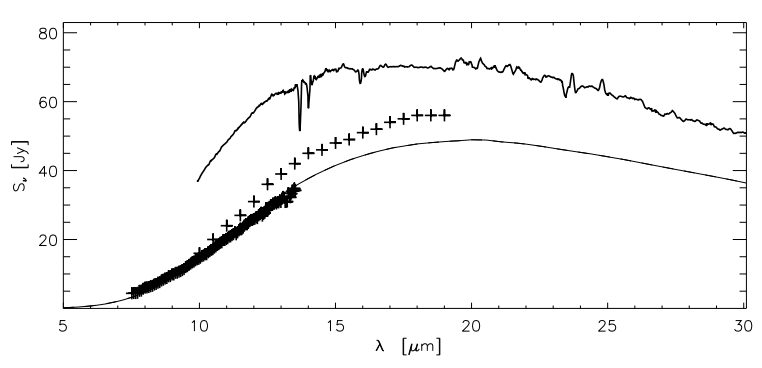

Fig. 1. MIDI spectrum (June 2007; thick crosses) compared with the Spitzer spectra obtained in April 2005 (upper solid curve, Evans et al. 2006) and May 2007 (crosses, Evans et al. 2009, in preparation). The smooth solid line is the flux from the best model assuming a distance of $3.1 \mathrm{kpc}$.

Simple models fail to account for the complex visibilities observed, although the difference between the mean level of the visibilities from the baseline UT2-UT3, and those obtained with UT3-UT4 suggest a flattened structure with a major axis orientation of approximately $110-140^{\circ}$. The differential phases point to a significant asymmetry in the direction of the minor axis, i.e. $\sim 45^{\circ}$.

We applied the continuum Monte Carlo radiative transfer code MC3D (Wolf 2003; Wolf et al. 1999), that solves the radiative transfer problem self-consistently. We use a classical model of a stratified disk of dust (Chesneau et al. 2006, 2007). The dust density follows a 2D law (both radial and vertical) given by:

$\rho(r, z)=\rho_{0}\left(\frac{R_{\star}}{r}\right)^{\alpha} \exp \left[-\frac{1}{2}\left(\frac{z}{h(r)}\right)^{2}\right]$

where $r$ is the radial distance in the midplane of the disk, $\alpha$ is the density parameter in the midplane, $R_{\star}$ is the stellar radius, and the disk scale height, $h(r)$, is given by $h(r)=h_{0}\left(r / R_{\star}\right)^{\beta}$, where $h_{0}$ is the scale height of reference, and $\beta$ is the vertical density parameter.

The absence of spectral features in the IR indicates that the dust is dominated by amorphous carbon grains. We assume the standard interstellar grain size distribution Mathis et al. (1977) with spherical grain radii extending from 0.005 to $1 \mu \mathrm{m}$. We estimate the outer radius of the disk to be $500 \mathrm{AU}$ consistent with an expansion since 1997 at a maximum velocity of $100 \mathrm{~km} \mathrm{~s}^{-1}$. The initial parameters ( $T_{\text {eff }}$ of the source and dust mass) were chosen following van Hoof et al. (2007). The outputs from the code are described in Chesneau et al. (2006, 2007). The distance, luminosity, and temperature of the central star are uncertain by a large factor (Jacoby et al. 1998). We first fixed the distance to be $1.5 \mathrm{kpc}$, but no satisfactory models could be found. 

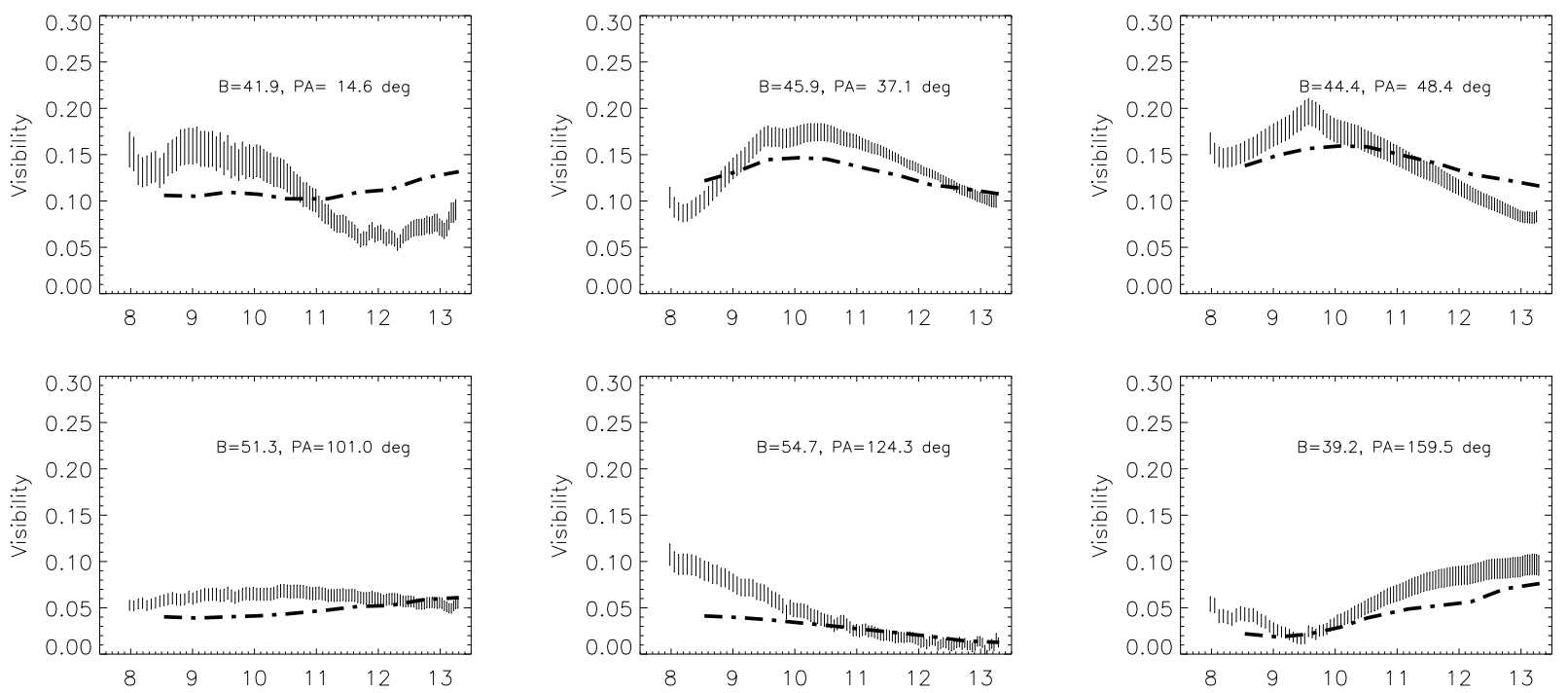

Fig. 2. MIDI visibilities with their error bars, compared with the best model fit (dashed-dotted lines, see Table 2). The $\chi^{2}$ values of the fits of 16.5, 7.1, 7.8, 7.6, 5.2 and 4.6. The reduced $\chi^{2}$ for the full data set is 8.2.

By increasing the distance, the fit of the visibility curves and the SED steadily improved and we achieved the best fit model for a distance of $3.5 \mathrm{kpc}$, a value close to the distance favored by van Hoof et al. (2007). However, we note that we did not explore the parameter space for larger distances and that the distance chosen is by no means a strong constraint. Our priority in this study was to derive the most consistent geometry of the source, by fitting the visibility curves. We were unable to find a model with a good match to both the visibility curves and the SED. The best model, presented here, is that which exhibits the highest flux although it is still too low. With slight modifications of the parameters, some models provided better fits to the visibilities (best $\chi^{2} \sim 6$ ), but a worse SED. Given the very short timescale to form the disk, and the large scale height inferred, a stratified disk might not be the best model to account for the density profile of the dust. A slowly expanding torus (Peretto et al. 2007) should be investigated in the future.

\section{Results and discussion}

The MIDI observations provide direct evidence for a thick, highly-inclined disk or torus (which therefore efficiently screens the central source), whose parameters are described in Table 2. The major axis orientation of the disk $\left(134 \pm 5^{\circ}\right)$ is in agreement with the asymmetry detected in the old PN $\left(130 \pm 8^{\circ}\right)$. A similar orientation is also mentioned in Kerber et al. (2002). Such an alignment between the disk and PN may not be fortuitous. In the case of V605 Aql, the disk and the planetary nebula A58 share the same major axis (Hinkle et al. 2008). Of importance too is the low level of asymmetry detected in the old PN compared to that observed in the disk. Is the new mass loss more asymmetric or is the smaller asymmetry of the PN remnant due to evolution and old age? Another related object worth mentioning is A 30, a PN that underwent its own VLTP a few thousand years ago (Borkowski et al. 1995). The inner nebula exhibits a thin equatorial disk, which is currently being eroded by the fast wind of the central star, in contrast to its old PN, perfectly round.

The disk is very optically thick and, at $8 \mu \mathrm{m}$, most of the light emerges from a small region above the North pole (see Fig. 4). The scale-height of the disk/torus is large, limiting the opening

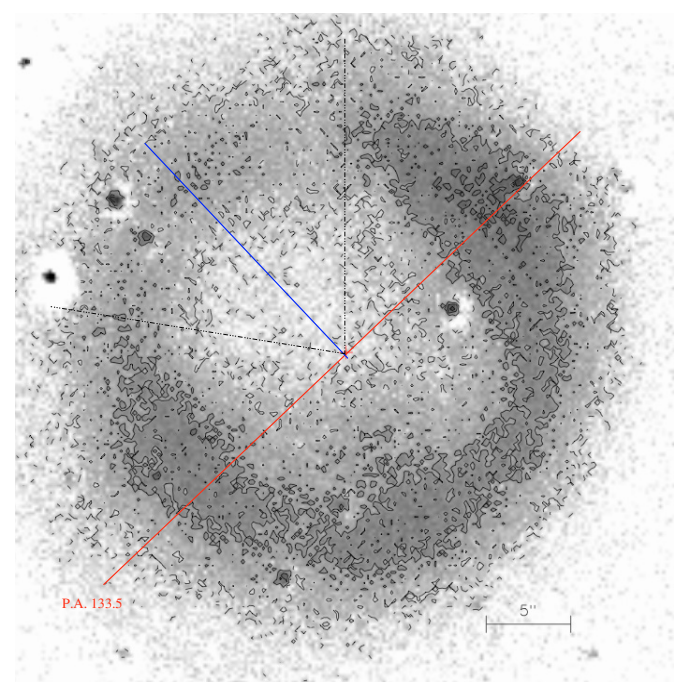

Fig. 3. Continuum subtracted [OIII] contour plot of the old PN recorded in 2002. The region oriented at $\mathrm{PA} \sim 130^{\circ}$ is brighter and a hole is visible in the North-East part of the roughly spherical nebula, with an halfaperture angle of $\sim 45^{\circ}$. North is up and East is left.

angle of the polar regions, which is reminiscent of the long-lived "wall" structure seen in the binary post-AGB HR 4049 (Dominik et al. 2003). Spherical models of the dust shell explain the shift of the peak of the SED toward longer wavelengths by suggesting that the inner boundary has receded from the star (Käufl et al. 2003; Evans et al. 2006). If this was related to a cessation of mass loss and dust formation, then the optical depth of dust would decline rapidly, assuming that the dust is moving radially away from the star. The estimated size of Sakurai's Object disk from our observations in 2007 is small compared with previous estimates, and implies that the high velocities inferred from spectral lines may be inconsistent with the dust expansion. If the expansion velocity were consistent with the rapid motions observed for $\mathrm{CO}$ and optical lines, the disk should already be resolvable by $8-\mathrm{m}$ class telescope in the infrared (Eyres et al. 2004; Kerber et al. 2002; Worters et al. 2008). 

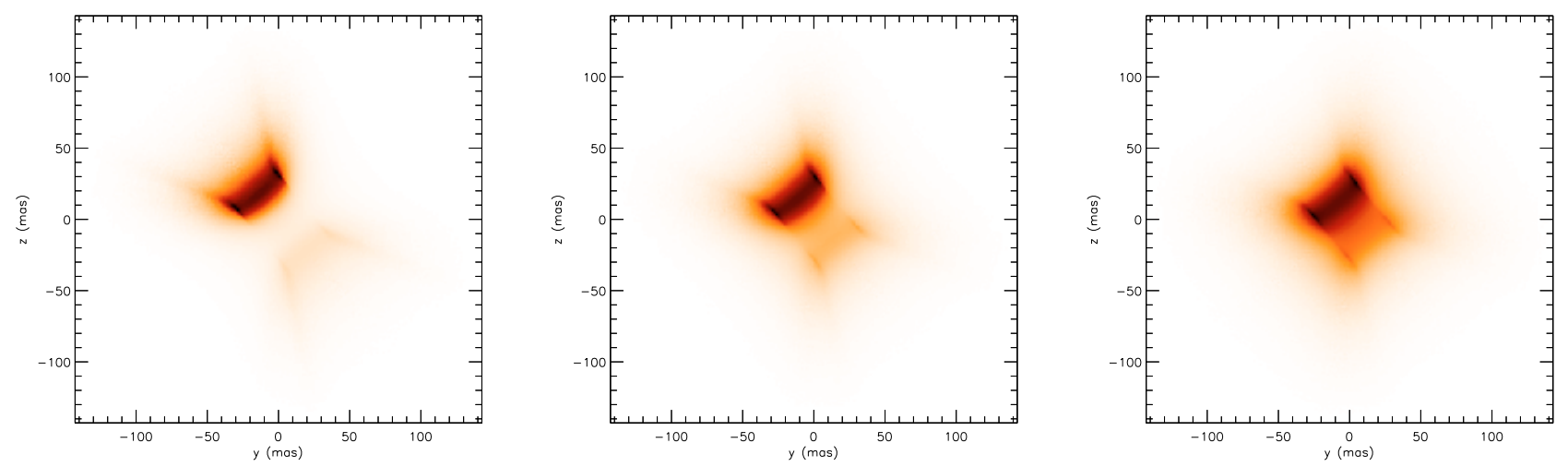

Fig. 4. Flux distribution of the model at $8,10.5$ and $13 \mu \mathrm{m}$. A small amount of light emerges at $8 \mu \mathrm{m}$ while the flux comes from deeper in the disk at $13 \mu \mathrm{m}$.

The fast spectral evolution of the source toward lower temperatures, as witnessed by the Spitzer and MIDI spectra, must be interpreted as a growing opacity of the disk, whether by accumulation of new material or by grain growth. Only the continuous production of new dust and/or grain growth can explain the shift of the SED of the disk toward longer wavelengths. A more rapid expansion of the disk as an explanation for the SED evolution has been ruled out by the smaller disk size reported here. Since the dust on the inner rim of the disk is close to the sublimation temperature, and the outer radius is not well constrained by the $N$-band measurements, a dust expansion rate cannot be accurately estimated. Keplerian motion (i.e. with a very low radial expansion) of the dust in the disk cannot be excluded.

The discovery of a flattened structure such as a disk is of great importance to the future interpretation of the outburst of Sakurai's Object and the VLTP phenomenon, as much of the previous work is based on the assumption of spherical ejection. Disk models may do a better job of fitting the observed parameters. A well-known consequence of applying a 1D radiativetransfer code in the dust to a 2D astronomical source is that the dust is put much farther than in reality, biasing strongly all the inferred parameters.

The formation of a disk/torus could be explained by two classes of models: those involving an intrinsic asymmetry of the ejection, and those involving a binary companion. The models should also account for the asymmetry seen in the surrounding PN which implies that the same asymmetry has been acting on the mass loss for $10000 \mathrm{yr}$. Binarity can naturally explain the disk-like geometry and also the fast wind features (such as the He I $\lambda 10830$ line and the hole at PA $\sim 45^{\circ}$ ). The companion would likely be a low-mass object which has survived the previous evolution of the system (Mullally et al. 2007). The fact that the asymmetry of the disk is much more pronounced in the recent VLTP event could be a consequence of the smaller mass of the ejecta. We emphasize that, in this scenario, the original ejection might have been spherical, but with the asymmetry being enhanced by the influence of the companion. The short timescale for disk formation is a severe constraint and may imply that the orbital period of the companion is short (i.e. typically a few months). Given their similarities, any solution to the asymmetry of the Sakurai's Object dust envelope will also apply to its twin, V605 Aql (Clayton et al. 2006). In both cases, there appears to be a thick disk of dust preventing the star from being seen directly. In both cases too, some structure in the surrounding $\mathrm{PNe}$ are aligned with the axis of the disk.
The low rate of expansion of the disk provides a strong support for a binary interaction. A VLTP occurs in 10-20\% of postAGB stars. A companion, originally distant from the primary ( 5-10 AU) so to avoid mass transfer, and a common envelope phase could be responsible for the outflow shaping. During the initial AGB phase, the spherical wind from the primary star interacts with the companion and is focused toward the equatorial plane. Initially, the asymmetry is strong. However, after $10^{4}$ years, the asymmetry slowly disappears as the nebula expands into the ISM. When the VLTP occurs, the resulting massloss also interacts with the companion and is once again focused towards the equatorial plane. Given the mass ejected, the companion might also migrate inward and may provide sufficient angular momentum to stabilise the ejecta in the form of a torus (Nordhaus \& Blackman 2006; Peretto et al. 2007; Edgar et al. 2008). Given the disk inclination and high optical depth, the detection of such a companion will be a difficult challenge for the foreseeable future.

\section{References}

Berger, L., Koester, D., Napiwotzki, R., et al. 2005, A\&A, 444, 565 Borkowski, K. J., Harrington, J. P., \& Tsvetanov, Z. I. 1995, ApJ, 449, L143 Chesneau, O., Collioud, A., De Marco, O., et al. 2006, A\&A, 455, 1009 Chesneau, O., Lykou, F., Balick, B., et al. 2007, A\&A, 473, L29 Clayton, G. C., \& De Marco, O. 1997, AJ, 114, 2679

Clayton, G. C., Kerber, F., Pirzkal, N., et al. 2006, ApJ, 646, L69 Dominik, C., Dullemond, C. P., Cami, J., et al. 2003, A\&A, 397, 595 Edgar, R. G., Nordhaus, J., Blackman, E. G., \& Frank, A. 2008, ApJ, 675, L101 Evans, A., Tyne, V. H., van Loon, J. T., et al. 2006, MNRAS, 373, L75 Eyres, S. P. S., Smalley, B., Geballe, T. R., et al. 1999, MNRAS, 307, L11 Eyres, S. P. S., Geballe, T. R., Tyne, V. H., et al. 2004, MNRAS, 350, L9 Hajduk, M., Zijlstra, A. A., Herwig, F., et al. 2005, Science, 308, 231 Herwig, F. 2001, ApJ, 554, L71

Hinkle, K. H., Lebzelter, T., Joyce, R. R., et al. 2008, A\&A, 479, 817 Jacoby, G. H., De Marco, O., \& Sawyer, D. G. 1998, AJ, 116, 1367 Käufl, H. U., Koller, J., \& Kerber, F. 2003, A\&A, 406, 981

Kerber, F., Köppen, J., Roth, M., \& Trager, S. C. 1999, A\&A, 344, L79 Kerber, F., Pirzkal, N., De Marco, O., et al. 2002, ApJ, 581, L39 Lawlor, T. M., \& MacDonald, J. 2003, ApJ, 583, 913 Leinert, C., Graser, U., Przygodda, F., et al. 2003, Ap\&SS, 286, 73 Mathis, J. S., Rumpl, W., \& Nordsieck, K. H. 1977, ApJ, 217, 425 Mullally, F., Kilic, M., Reach, W. T., et al. 2007, ApJS, 171, 206 Nordhaus, J., \& Blackman, E. G. 2006, MNRAS, 370, 2004 Peretto, N., Fuller, G., Zijlstra, A., \& Patel, N. 2007, A\&A, 473, 207 Ratzka, T., Leinert, C., Henning, T., et al. 2007, A\&A, 471, 173 Tyne, V. H., Evans, A., Geballe, T. R., et al. 2002, MNRAS, 334, 875 van Hoof, P. A. M., Hajduk, M., Zijlstra, A. A., et al. 2007, A\&A, 471, L9 Wolf, S., Henning, Th., \& Stecklum, B. 1999, A\&A, 349, 839 Wolf, S., Padgett, D. L., \& Stapelfeldt, K. R. 2003, ApJ, 588, 373 Worters, H., Rushton, M. T., et al. 2008 [arXiv:0810.4556] 